\title{
Rituximab is safe and effective in FRNS and SDNS -but where to go from here?
}

\begin{abstract}
Although the anti-CD20 B-cell depleting biological agent rituximab has been shown in small series and single-arm studies to effectively treat frequently relapsing nephrotic syndrome (FRNS) and steroid-dependent nephrotic syndrome (SDNS), only now has a multicentre, double-blind, randomized, placebo-controlled trial been reported with $>3$ months follow-up data. The results, newly published in The Lancet, show that rituximab is safe and effective in patients diagnosed with childhood-onset nephrotic syndrome.
\end{abstract}

The vast majority of patients with idiopathic nephrotic syndrome are treated in the first line with steroids. However, for those who experience more than four relapses or who relapse at least twice within 6 months of remission (FRNS) and those who relapse once steroids are discontinued or tapered (SDNS), immunosuppressive treatments with ciclosporin, cyclophosphamide, mizoribine and mycophenolate mofetil are used. "Unfortunately, 10-20\% of children have so-called complicated FRNS or SDNS-showing frequent relapses or steroid dependency while on immunosuppressants," explains lead investigator Kazumoto Iijima. Furthermore, immunosuppressants (for example, ciclosporin) are associated with nephrotoxicity that limits chronic use. "New treatments that do not involve steroids or immunosuppressive agents for these conditions are urgently needed."

Rituximab was introduced into clinical care as a second-line treatment for nephrotic syndrome when the 2012 Kidney Disease: Improving Global Outcomes (KDIGO) Clinical Practice Guideline recommended the agent for childhood-onset complicated FRNS and SDNS, conditions in which B-cell immunity is altered. Despite this recommendation, high-level clinical data supporting its safety and efficacy were lacking. Iijima and colleagues conducted their trial in 48 patients, who were randomly assigned (1:1) to either rituximab or placebo. Patients in the experimental arm received a weekly intravenous dose of $375 \mathrm{mg} / \mathrm{m}^{2}$ (dosing based on recommendations in B-cell lymphoma) for 4 weeks. At the time of assignment, all patients received standard steroid treatment (prednisolone); all immunosuppressants were stopped 169 days after assignment. The primary end point was the relapse-free duration, and the follow-up period was 1 year.

Patients in the rituximab group had longer relapse-free durations (median 267 days; 95\% CI 223-374) than those in the placebo group (median 101 days; 95\% CI 70-155). Although promising, at the end of the follow-up period a considerable proportion of the patients in the rituximab group (71\%) had relapsedand all patients in the trial had relapsed by 19 months. "To extend the relapsefree period further, modification of the rituximab regimen-and, possibly, adjunct immunosuppressive therapies - might be necessary," considers Iijima.

Overall, $42 \%$ of patients in the rituximab group and $83 \%$ in the placebo group had treatment failure, which was defined as relapse by day 85 , a diagnosis of FRNS or SDNS between days 86 and 365 or development of steroid resistance. "When the patients developed treatment failures, their allocation code was urgently disclosed. If the patients were given placebo, they were able to enter the separately conducted rituximab pharmacokinetic study," explains Iijimia. This study design meant that all participants could continue in the study.

Although the number of infectious events was higher in the rituximab group than the placebo group, the infections were mild and treatable. The most common adverse events in the treatment arm were hypoproteinaemia,

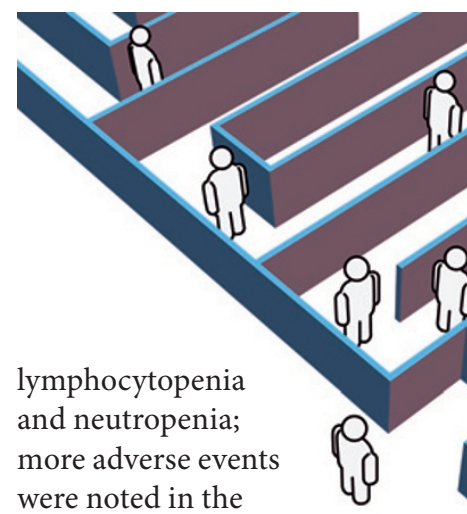

experimental arm,

but the difference did not reach statistical significance. "Our study provides the first firm evidence that rituximab is safe and effective-at least for 1 year-for the treatment of childhood-onset complicated FRNS and SDNS," says Iijima.

These results support the continued investigation of rituximab in childhood FRNS and SDNS. However, many questions remain unanswered. Rituximab does not 'cure' these conditions, and repeated courses might be necessary. Given this possibility, the long-term effects of rituximab use must be determined, particularly in children. As the authors point out, although a long steroid-free period after rituximab treatment can potentially enable patients to recover from adverse effects, such as impaired growth, longer-term monitoring is needed to determine if this is the case.

Iijima and his team intend to continue their studies with "comparisons of the efficacy, safety and cost-effectiveness of various rituximab dosing regimens and B-cell driven regimens."

Mina Razzak

Original article lijima, K. et al. Rituximab for childhood-
onset, complicated, frequently relapsing nephrotic
syndrome or steroid-dependent nephrotic syndrome:
a multicentre, double-blind, randomised, placebo-controlled
trial. Lancet doi:10.1016/S0140-6736(14)60541-9

